\title{
PLANES DE IGUALDAD EN LAS UNIVERSIDADES ESPAÑOLAS. ANÁLISIS DE CONTENIDO DESDE LAS CIENCIAS SOCIALES
}

\author{
EQUALITY PLANS IN SPANISH UNIVERSITIES. CONTENT ANALYSIS \\ FROM THE SOCIAL SCIENCES
}

Francisco de los Cobos Arteaga

Universidad de Castilla-La Mancha, Toledo. España/Spain

Fco.Cobos@uclm.es

Recibido/Received: 30/07/2012

Aceptado/Accepted: 27/09/2012

\begin{abstract}
RESUMEN
En este artículo, desde la visión de las ciencias sociales, se evalúa la aptitud científica de los planes de igualdad redactados en las universidades españolas para cumplir con las leyes de igualdad de mujeres y hombres, con especial atención al profesorado. La metodología empleada es el análisis cualitativo de los planes mediante la identificación de unidades de registro definidas en la revisión teórica del objeto de estudio. Como principales resultados se evidencia un bajo compromiso de la universidad española con la igualdad y, a la hora de elaborar el plan, carencias de conocimientos en ciencias sociales y teoría feminista, que son las disciplinas en cuyo núcleo se ha desarrollado los conceptos de género y la igualdad.
\end{abstract}

\section{PALABRAS CLAVE:}

Igualdad, estratificación social, brecha de género, feminismo, universidad.

\section{SUMARIO}

1. Introducción. 2. El contexto de la igualdad en la sociedad española. 3. ¿Nuevas segregaciones verticales? De la reproducción patriarcal académica a la reproducción de élites familiares, de clase y religiosas. 4. La voluntad de desarrollar políticas de igualdad en el profesorado universitario. 5. Metodología de la investigación. 6. Discusión de los datos. 7. Reflexiones finales. Bibliografía.

\begin{abstract}
In this article, from the vision of the social sciences, there is evaluated the scientific aptitude of the plans of equality written in the Spanish universities to expire with the laws of equality of women and men, with special attention to the professorship. The used methodology is the qualitative analysis of the plans by means of the identification of units of record defined in the theoretical review of the object of study. As principal results there is demonstrated a low commitment of the Spanish university by the equality and, at the moment of elaborates the plan, lacks of knowledge in social sciences and theory feminist, that they are the disciplines in whose core has developed the concepts of kind and the equality.
\end{abstract}

\section{KEYWORDS:}

Social equality, social stratification, gender gap, feminism, university. 


\section{CONTENTS}

1. Introduction. 2. Context of equality in Spanish society. 3. New vertical segregations? From de patriarchal playback to academic playback, family, class and religious elites. 4 . The desire to develop equality policies in the university teaching staff. 5. Research methodology. 6. Discussion of the data. 7. Final thoughts. References.

\section{INTRODUCCIÓN}

La presente investigación tiene por objeto de estudio evaluar la calidad de los planes de igualdad que han debido redactarse en las universidades españolas para cumplir con las leyes de igualdad de mujeres y hombres. Con especial atención al profesorado, el análisis se realiza desde ciencias sociales, porque son estas disciplinas las que contemplan en sus conocimientos la igualdad y el género. Sobre la dimensión temporal del estudio, debe precisarse que, desde algunas instancias, se ha considerado como punto de inflexión en las políticas de igualdad el año 2007, al coincidir en esta fecha la promulgación de un conjunto de disposiciones normativas -Ley Orgánica 3/2007, de 22 de marzo, para la igualdad efectiva de mujeres y hombres, Ley Orgánica 4/2007, de 12 de abril, por la que se modifica la Ley Orgánica 6/2001 de Universidades y la Ley 7/2007, publicada en la misma fecha, del Estatuto Básico del Empleado Público. Si bien, para ofrecer una visión científica, el periodo de estudio debe remontarse varias décadas, porque la igualdad como principio vertebrador de la sociedad quedó establecida en el artículo 1.1. de la Constitución Española de 1978.

En todo caso, conforme con la exposición de motivos de la Ley Orgánica 3/2007: “el reconocimiento de la igualdad formal ante la ley, aun habiendo comportado, sin duda, un paso decisivo, ha resultado insuficiente". Por eso, para superar esta carencia, la misma norma ordena una sucesión de trabajos a las universidades. En primer lugar, deben elaborar planes de Igualdad: "un conjunto ordenado de medidas adoptadas después de realizar un diagnóstico de situación, tendentes a alcanzar en la empresa la igualdad de trato y de oportunidades entre mujeres y hombres y a eliminar la discriminación por razón de sexo". Paso inicial que, en correspondencia con el carácter científico de las instituciones académicas, tiene que ser llevado a cabo de acuerdo con los conceptos y categorías analíticas de los saberes en cuyo objeto de estudio tiene un lugar destacado las desigualdades sociales y sus variables explicativas. Una vez completada esta fase, la ley es muy precisa cuando determina que los contenidos de los planes deben: "fijar los objetivos concretos de igualdad a alcanzar, las estrategias y prácticas a adoptar para su consecución, así como el establecimiento de sistemas eficaces de seguimiento y evaluación de los objetivos fijados". Es evidente que, en sintonía con el carácter democrático de la institución universitaria, debe promoverse un amplio debate entre sus miembros, para decidir qué medidas y qué medios deben ser empleados para lograr la igualdad. Finalmente, la Ley exige transparencia en la "implantación del plan, garantizándose el acceso de la representación legal del personal al contenido de los planes y a la consecución de sus objetivos". Es decir, como en cualquier actividad académica, debe procederse a una evaluación -lo más objetiva y participativa posible- para conocer qué objetivos han sido alcanzados y qué medidas deben ser corregidas con el propósito de ser logradas en el futuro. 
Otro de los objetivos de este trabajo, con las limitaciones inherentes a la extensión de un artículo, es pretender aportar instrumentos con validez desde las ciencias sociales para evaluar de forma científica y, al mismo tiempo, comprensible el grado de cumplimiento de los planes de igualdad en las universidades. La originalidad de la investigación radica en el hecho que, hasta el momento, la literatura científica sobre evaluación de políticas universitarias en igualdad es limitada. Disponemos de un notable y pionero análisis del Grup d'Estudis sobre Sentiments, Emocions i Societat del Departament de Sociologia de la Universitat Autónoma de Barcelona (Izquierdo, 2004) y, tras la promulgación de la ley, debe destacarse el trabajo de las profesoras de sociología de la Universidad de Deusto (Elizondo, Novo y Silvestre, 2010). Asimismo, hay otro artículo que examina la repercusión de las políticas de género en la Universidad de Málaga (Morales, Luna Ji y Esteban, 2010). En definitiva, publicaciones valiosas, pero todavía insuficientes.

Tras esta introducción que delimita el objeto de estudio, el texto prosigue con un breve acercamiento al contexto de la igualdad en la sociedad española. El tercer punto se dedica a un problema a considerar en futuras investigaciones ¿el patriarcado académico puede seguir reproduciendo élites, ya no solo masculinas, sino sobre la base de la familia, la clase social o las hermandades religiosas? A continuación, como evidencia de la voluntad de desarrollar políticas de igualdad, se identifican los distintos puntos temporales en los que comenzó el camino por la igualdad en la universidad española y que han conformado, con relación al tema de la igualdad, una tipología de gobierno universitario. El quinto apartado está centrado en describir la metodología cualitativa de análisis de contenido del presente artículo. Ocupan los hallazgos de la investigación las siguientes páginas y, por último, se ofrecen las conclusiones del trabajo.

\section{EL CONTEXTO DE LA IGUALDAD EN LA SOCIEDAD ESPAÑOLA}

Todo estudio universitario sobre la igualdad de mujeres y hombres debería comenzar con el reconocimiento a quienes, tildadas de utópicas o avanzadas a su tiempo, abrieron esta vía a la sociedad. Asimismo, por evidenciar que, si hay un distintivo que dio unidad a estas pioneras del activismo y del pensamiento por la igualdad, es la fortísima contestación que recibieron por quienes apelaban al "carácter natural" de sus privilegios de gobierno, económicos, religiosos, sexuales y simbólicos. La génesis de esta discriminación, la existencia o no de un "contrato sexual" primigenio y autónomo respecto a otras desigualdades sociales (Pateman, 1995), excede el propósito de este trabajo. Lo que pretendemos que forme parte de esta reflexión es el hecho que, desde el mismo momento de visualizarse las reivindicaciones de emancipación de las mujeres, el poder definió el feminismo como una teoría de conflicto y legitimó, contra las demandas igualitarias, prácticas de "cierre social” (Parkin, 1984).

Una vez trazada esta línea entre el poder y el pensamiento de la igualdad, también debe significarse que, en el caso español se alcanzó el mismo trato legal de mujeres y hombres como "normalización" de un proceso ya logrado en las sociedades que son nuestros referentes culturales. Una primera fase, en la que las europeas habían conseguido igualdades formales, plasmadas en leyes para todas: derechos de propiedad, políticos, de acceso a la educación, al matrimonio y a decidir con libertad sobre su capacidad biológica de reproducción, como señalaba (Goldstein, 1982) en un texto fechado en Francia hace tres décadas. Por otra parte, estos logros formales, sin duda importantes, tienen su centro en el 
concepto de "empoderamiento". Una idea capital para la construcción de la identidad femenina contemporánea, que apela a la capacidad individualizada de la mujer, tanto en su vida personal, como en la inclusión en distintos ámbitos de la vida pública. Pero, una idea que, en muchos de sus escritos, considera al conjunto de las mujeres como un todo homogéneo dotado de las mismas oportunidades vitales.

Sirva la conocida frase de (Bourdieu 1999) "sin movimiento social, no hay política social", para evidenciar que la llamada "revolución silenciosa" (Inglehart, 1977) introductora de cambios en los estilos de vida de las nuevas generaciones-, no ha conseguido todavía provocar una crisis de legitimación del patriarcado en España. Los bajos niveles de asociacionismo en partidos políticos y sindicatos y las diferencias entre los géneros a hora de concebir sus vínculos cívicos, pueden ser las causas explicativas de este fracaso, de acuerdo con (Morán, 2011). Como resultado de estas carencias, en los países del sur de Europa se ha consolidado una particular estructura social denominada en la literatura científica desde (Esping-Andersen, 1990): "familismo", "de beneficencia", "sureña" o "patriarcal". Una estructura que determina una constante en las decisiones políticas españolas, caracterizadas por bajas dotaciones de las administraciones públicas, respecto a los países europeos, en educación y en los ámbitos presupuestarios clasificados bajo "protección social" -salud, discapacidad, pensiones, familia, infancia y vejez, desempleo, vivienda y exclusión social-, como puede comprobarse en las largas series ofrecidas por (Eurostat, 2009), y que ha sido reiteradamente evidenciado, entre otras y otros, por (Navarro, 2008), (Poveda, 2011) y (Alguacil, 2012). Por supuesto, como mostró hace tiempo María Ángeles Durán con los estudios sobre el uso de tiempo y los costes invisibles de los cuidados, estas políticas se realizan con el convencimiento que el estado de bienestar descansa en el trabajo altruista e invisualizado de las mujeres en el seno de la familia heteropatriarcal (Durán, 1996, 1999, 2000, 2006).

Es evidente que, estas economías realizadas por los gobiernos han tenido consecuencias negativas, tanto para las sociedades asentadas sobre estas prácticas como para las mujeres, porque el declive del modelo familiar del varón como único sustentador económico no ha sido acompañado de un reemplazo progresivo de los hombres, ni de las administraciones públicas en las tareas de cuidados. (González y Jurado, 2009). Un conjunto de desigualdades institucionalizadas, que afecta decisivamente a las profesoras, manifestándose en una segregación vertical en el empleo o "techo de cristal", a la hora de consolidarse y promocionar en la universidad (Callejo, 2004) y (Canino y Vessuri, 2008). Una vez reconocido el problema de los techos de cristal, es necesario evidenciar que para ser eliminados hay un conflicto manifiesto entre quienes, por una parte, patrocinan las teorías funcionalistas de la meritocracia credencialista como mecanismo para la igualdad de oportunidades (Collins, 1979) y, por en otro lado, las teorías educativas de la reproducción y resistencia que muestran el proceso de enseñanza como decisivo para mantener las relaciones de clase, de género y culturales -Parkin, Bourdieu, Passeron, Bernstein, Apple...-. Con respecto a sus hallazgos, hay suficiente consenso en la sociología de la educación cuando se precisa que el conocimiento es transmitido tanto a través del aprendizaje de conceptos, como de valores sociales y que, estos últimos, se adquieren de forma parcialmente inconsciente, recibiendo la denominación de "currículum oculto" (Apple, 1986).

También, se ha señalado que, si bien los conceptos a transmitir en la docencia son determinados por la ideología dominante, los valores no manifiestos son el fundamento de la coerción simbólica sobre quienes aprenden, para que estos internalicen los esquemas de 
percepción y acción correspondientes a los intereses materiales y simbólicos de los grupos del poder (Bourdieu y Passeron, 1985), (Bernstein, 1988) y (Bourdieu y Wacquant, 1995). Nada más innegable que los datos empíricos para validar las teorías de la reproducción, como puede comprobarse desde el mismo momento que las mujeres y los hombres "eligen" distintas opciones educativas. En un porcentaje superior, los varones son "dirigidos" al éxito profesional y las "preferencias" de las mujeres son "orientadas" a garantizar que se reproduzca de manera adecuada el "orden natural", conformándose una primera segregación horizontal por los hábitos atribuidos culturalmente a cada género. Poco cambia, cuando concluye el proceso formativo dirigido por las pautas meritocráticas. Las posiciones iniciales de clase, género y culturales se trasladan, casi de forma íntegra, al mercado laboral en tasas de actividad, paro, precariedad, percepciones económicas y espacios de poder, desiguales entre mujeres y hombres, pobres y pudientes y quienes participan o no de los supuestos centrales de la cultura. En consecuencia, a nadie en el pensamiento feminista le es ajena la expresión "lo personal es político" para evidenciar el vínculo estrecho entre los invisibles trabajos del hogar, que recaen mayoritariamente sobre el grupo de las mujeres, la educación como prolongación de las "responsabilidades" del cuidado doméstico" y, por último, el trabajo remunerado como visualización económica y de poder. Al respecto, se recomienda la lectura de la excelente edición de artículos de la politóloga (Showstarkc, 1996).

\section{3. ¿NUEVAS SEGREGACIONES VERTICALES? DE LA REPRODUCCIÓN PATRIARCAL ACADÉMICA A LA REPRODUCCIÓN DE ÉLITES FAMILIARES, DE CLASE Y RELIGIOSAS}

A principios de los noventa, con la expresión "élites discriminadas" se hacía referencia a las profesoras pioneras que debían hacer esfuerzos suplementarios con relación a los varones para ser reconocidas como académicas por sus pares (García de León, 1990). Una posición intelectual y ante el patriarcado, que nadie mejor que (Woolf, 2008) expresó con la sentencia que las mujeres precisaban una "habitación propia" -espacio y tiempo para desarrollarse autónomamente-. Transcurridas dos décadas, como reflejo de la reducida población activa femenina, en la universidad española todavía hay pocas profesoras y concentradas en las llamadas disciplinas blandas, cualitativas, con saberes sujetos a debate público, y construidas en las representaciones sociales como prolongación de las tareas domésticas femeninas, que el pensamiento tradicionalista continúa atribuyendo a las mujeres. Frente a estos conocimientos, las ciencias duras, fuertes, exactas, cuantitativas o profesionalizantes de varones (Fernández, 2008). Además, de esta segregación horizontal en términos de género, una vez iniciada su carrera en la universidad el profesorado adquiere la cognición que, para poder progresar en el sistema de la enseñanza superior, debe orientar su actividad investigadora para transferirla utilitariamente a beneficio propio que, con asiduidad, coincide con el poder académico. (Díez, 2009). Definitivamente, como en cualquier proceso de aprendizaje, después de una exposición reiterada a estas normas simbólicas, la mayoría de los partícipes en este proceso "interiorizan" los valores del poder universitario como correctos. Unos valores, en buena medida, coincidentes con los roles tradicionales masculinos. (Sánchez Bello, 2002) y (Fernández Rius, 2008).

Cuando acercamos nuestra atención a la normativa de igualdad universitaria de 2007, si hay una medida exitosa es la exigencia que hace de componer de forma equilibrada los 
órganos y cargos de responsabilidad, con la justificación que la presencia de mujeres en altos puestos constituye un ejemplo de empoderamiento para las nuevas generaciones (Carlos, 2007). Si bien, es evidente que, las ciencias sociales no pueden tomar en consideración a un agente aislado y dotado de un interés exclusivamente propio, porque ya se ha mostrado que, en general, los individuos operan conforme a las recompensas del poder. En este sentido, el estudio científico de los grupos evidencia como axioma que desde las familias se desarrollan complejas estrategias de reproducción, no necesariamente conscientes y racionales, sino impulsadas por el habitus (Bourdieu, 2004). Y, además, estas estrategias se utilizan para mantener a cierta clase social en el poder y para excluir a otros grupos económicos, de género, étnicos y culturales de los instrumentos del poder (Chomsky y Foucault, 2006).

Sirvan estos argumentos, para volver a interrogarse en el sentido de los excelentes análisis de (García de León, 2001), que ponían énfasis en la disposición de "capital cultural" de las profesoras pioneras en España, un hábito proporcionado por el ambiente de sus familias que, ya insertadas en la academia, facilitó el acceso de esas profesoras. En el mismo sentido, a ningún veterano participante del mundo universitario le han sido ajenos los mecanismos de cooptación de profesorado sobre las bases de la cultura de departamento, la familia, la clase social o las hermandades masculinas y religiosas (Cruz y Sanz, 2010). Ante estos mecanismos endogámicos, las agencias de acreditación, las comisiones evaluadoras de la actividad investigadora y las leyes de igualdad entre mujeres y hombres de 2007 han pretendido establecer formalmente criterios "universalistas" en el acceso y promoción de las personas dedicadas a la universidad. En todo caso, y a modo de hipótesis para futuros estudios, debe considerarse que la investigación, tal y como está definida en el presente sistema universitario mediante su ejercicio en equipos, aunque permite incorporar diversas perspectivas, refuerza la estructura jerarquizada y patriarcal del sistema universitario. No parece menos cierto, que los llamados criterios "universalistas" introducen prácticas extrañas a las ciencias sociales y humanidades, campos en los cuales es difícil concebir el trabajo imaginativo, minucioso y solitario de las grandes pensadoras en un burocratizado grupo de investigación sujeto a la búsqueda de subvenciones y al relleno de formularios para agencias evaluadoras con normas definidas por empresas que indexan conocimientos.

Finalmente, parece oportuno clarificar a quienes son ajenos a la universidad que, a diferencia de otros trabajos, en la educación superior transcurren los plazos para el reconocimiento de la calidad docente, de la actividad investigadora o para las acreditaciones decisivas en la estabilidad o promoción del profesorado, sin poder ser detenidos sus vencimientos por enfermedades personales, ni por las tareas de cuidados o por la opción legítima de las mujeres a ser madres. Por tanto, en un sector donde está admitido el principio de la flexibilidad horaria sobre la jornada máxima y los días de descanso, quienes están sometidos a las obligaciones culturales o impuestas de cuidados, carecen de apoyos familiares o de capacidad económica para externalizarlos del hogar, no disponen de las mismas oportunidades en los constantes procesos de evaluación que son intrínsecos a la actual universidad. Como resumen, coincidimos con (Jiménez, Álvarez, Gill, Murga y Téllez, 2006) y (Morán, 2011) en señalar que la igualdad de género está más desarrollada en las sociedades más ricas y seguras y entre los grupos más ricos, más educados y que gozan de mayor estabilidad. Y, definitivamente, con (Gill, 2009) y (Cobo, 2011) cuando plantean que los dos sistemas hegemónicos occidentales -capitalismo 
neoliberal y patriarcado-, han pactado nuevos espacios para el doble trabajo de las mujeres. Entre ellos, la precarizada universidad española.

\section{LA VOLUNTAD DE DESARROLLAR POLÍTICAS DE IGUALDAD EN EL PROFESORADO UNIVERSITARIO}

Antes de dar cuenta de la metodología de la investigación, debe ser advertida la persona lectora que, por la insuficiente literatura especializada sobre el impacto de las políticas de igualdad en la enseñanza superior española, a modo de hipótesis, solo se pretende realizar una aproximación a la voluntad de desarrollarlas. En principio, debe significarse que en el tratamiento del género y de la igualdad se ha partido desde dos puntos temporales, en correspondencia a dos formas de conocimiento académico muy diferentes. Desde hace décadas, un reducido número de docentes de las universidades públicas, como parte de sus clases e investigaciones, crearon y transmitieron saberes científicos sobre estos temas y, además, ejercieron su activismo en la sociedad. Debe reconocerse a estas pioneras feministas, que sus iniciativas se desarrollaron de forma "no institucional $\mathrm{y}$, en numerosas ocasiones, con la decidida oposición de compañeros partidarios del "orden natural". Si bien, tras cursos de lucha, los movimientos sociales que impulsaron tuvieron cierta repercusión en la política social de sus universidades. En un segundo término, el interés por estos temas de algunos docentes y equipos rectorales comenzó a raíz de la Ley Orgánica 4/2007. Y, en último lugar, el hecho más destacable es la constatación de que, un lustro después de ser promulgado el derecho antidiscriminatorio contra las mujeres en la enseñanza, la mayoría de las universidades no han comenzado a caminar en el proceso de igualdad o no han completado aún su trabajo.

Por tanto, debemos reconocer que, en el momento presente, el logro de la igualdad de mujeres y hombres sigue jalonado por múltiples escollos. El primero, el todavía desconocimiento de buena parte de las autoridades académicas de unos contenidos y de un conjunto de valores centrales para la sociedad española como los que aporta la igualdad, que incluso dispone de un ministerio para su desarrollo. Tampoco es ajena a esta resistencia al cambio, la reproducción de relaciones internas de poder en las universidades que, con frecuencia, limitan estos temas o incluso los obvian a beneficio de intereses consolidados por la tradición de cada universidad. El último escollo es el más preocupante $\mathrm{y}$, como reconoceremos en la investigación, más arraigado, porque muchas instituciones de enseñanza superior, por falta de cultura política, infravaloran la igualdad entre mujeres y hombres y priorizan otros temas. En todo caso, conviene dejar claro que en las universidades españolas, cuya autoridad superior se elige mediante voto segmentado por estamentos, en la decisión o inacción final de implementar políticas de igualdad es decisiva la voluntad ideológica de sus equipos de gobierno; si bien, esta voluntad es orientada por la composición de fuerzas de los movimientos sociales que se manifiestan en la propia institución.

Una vez examinados las vías académicas hacia la igualdad, puede esbozarse una tipología de gobierno universitario: 1) Comprometido con la igualdad, a partir del trabajo científico y de los movimientos sociales gestados en el interior de la universidad, 2) Cumplidor burocrático de la ley, y 3) Esquivo por desconocimiento, carencia de de cultura política, incapacidad o minusvaloración. Además, estos estilos de gobierno, implican formas muy diferentes de abordar el conocimiento. El primer tipo, cuenta con trabajos realizados 
desde las ciencias sociales, porque los conceptos de género e igualdad implican, necesariamente, contemplar las categorías analíticas que son propias de estos ámbitos del conocimiento. Y, por último, un estilo de gobierno centrado en el cumplimiento burocrático de las leyes, que con dificultad puede avanzar más allá de reconocer diferencias estratificadas entre personas y grupos sociales. Un estilo que, a la hora de la verdad, mantiene las "reglas neutrales", formales o jurídicas $\mathrm{y}$, en este sentido, poco aporta a la hora de definir e implementar políticas reales de igualdad.

\section{METODOLOGÍA DE LA INVESTIGACIÓN}

El universo de la presente investigación está constituido por las 75 universidades de nuestro país, 51 públicas y 24 privadas, recogidas en el directorio del Ministerio de Educación, Cultura y Deporte. Secretaria de Educación, Formación Profesional y Universidades que, asimismo, ofrece enlaces a cada una de las instituciones. La metodología utilizada en este trabajo es el análisis de contenido cualitativo de los planes de igualdad, mediante el programa de tratamiento de datos Atlas.ti, definiéndose las unidades de registro mediante "palabras clave" -en los idiomas de redacción de los planes: castellano, catalán y gallego-. Con estas palabras, se pretende recoger los conceptos claves de las ciencias sociales y los, comúnmente, admitidos en el pensamiento feminista que, en cierta medida, deben estar contenidos en los planes como reflejo de los conocimientos de las personas redactoras. Es necesario significar que, estos conceptos han sido suficientemente "normalizados" con su inclusión en glosarios en los planes de igualdad de algunas universidades, con objeto de ser consultados por las personas que no disponen de suficientes nociones sobre el tema.

Respecto al procedimiento de recogida de datos, la consulta de los planes se realizó en el mes de marzo de 2012, fecha en la que se cumplieron cinco años desde la entrada en vigor de la normativa, un periodo lo suficiente dilatado para que las universidades hubiesen completado sus trabajos de diagnóstico y elaboración de planes de igualdad. Con relación al acceso a los datos de las instituciones superiores de enseñanza, se ha considerado que debía ser través de la consulta de sus páginas web, el mismo procedimiento mediante el cual puede acceder toda la ciudadanía. El criterio utilizado para determinar que una universidad enfatiza la igualdad dentro de su oferta formativa, es su localización desde un enlace ubicado en la página principal de la universidad o mediante el buscador que se dispone en la citada página. Para recopilar este dato, la persona encargada de esta tarea fue instruida para realizar la exploración con estos criterios y tuvo un tiempo asignado de 10 minutos para localizar cada plan. Posteriormente, quien suscribe el texto efectuó una segunda búsqueda y se comprobaron las discrepancias entre las dos recopilaciones. En todo caso, de ninguna manera debe interpretarse que la falta de consideración del plan de una universidad, signifique que la institución carezca del mismo. Solo, que no ha sido destacado suficientemente en su oferta ante la comunidad.

El instrumento de medida ha sido definido a través de tres grupos de categorías temáticas, que incluyen siete unidades de registro contextualizadas en frases y codificadas de forma dicotómica ordinal. Después de ser identificadas, estas medidas se cuantifican a través de frecuencias simples. Para lograr una valoración positiva, el texto deberá contener, al menos tres veces la palabra clave contextualizada. A continuación, en los grupos dos y tres de categorías temáticas, son señaladas entre guiones y en cursiva estas palabras clave. 
1. Importancia del Plan de Igualdad en el conjunto de la oferta universitaria. Forma parte de las señas de identidad de toda organización destacar selectivamente los aspectos que pretenden transmitir a la comunidad. Por eso, en la heterogénea enseñanza española puede localizarse un amplio abanico de actividades que son acentuadas, desde sus páginas web, como parte del atractivo singular de cada institución académica para reclutar estudiantes: idiomas, prácticas empresariales, intercambios con el extranjero, voluntariado, pastoral, deportes... e igualdad. También, se estiman positivas las menciones en los planes a la trayectoria académica y a la capacidad de generar impulsos comunitarios en el tema de la igualdad. Por contrario, si el camino permanece inconcluso o no es visible, se considera un compromiso insuficiente con la igualdad.

2. Plan redactado conforme con los criterios de las ciencias que tienen por objeto de estudio la igualdad y el género. Todas las acciones universitarias, en correspondencia con los objetivos atribuidos socialmente a su profesorado y a los equipos de gobierno, deben estar orientadas por criterios científicos. Por tanto, en este grupo se valoran dos unidades de registro. En primer lugar, si el plan está elaborado conforme a las ciencias sociales -social-, disciplinas en cuyo objeto de estudio tiene un papel destacado la igualdad y el género. En segundo término, como en cualquier texto académico, el plan debe incluir conceptos y referencias teóricas y/o empíricas, contrastadas en la investigación universitaria; si bien, el término -transversalidad-mainstreaming-, puede ser una palabra clave de consenso entre los planes científicos y aquellos conducidos por criterios administrativos o empresariales que, intuitivamente, contemplan la igualdad.

3. Determina el Plan las acciones a realizar, quiénes son las entidades o personas responsables de llevarlas a cabo, los mecanismos para negociar con la representación laboral y cómo evaluar la consecución de los objetivos. Estas unidades de registro tienen por objeto medir el grado de compromiso institucional de la universidad con relación a la igualdad, de acuerdo a: 1) ¿el plan incluye -acciones- susceptibles de ser llevadas a cabo por la universidad?; 2) ¿se identifican las entidades y las personas -responsables- de llevar a la práctica estas medidas?; 3) ¿las acciones son consensuadas con el profesorado, el personal de administración y servicios o, como impone la ley, con los -sindicatos- para garantizar una implantación democrática? y, por último, 4) ¿hay mecanismos objetivos y públicos de -evaluación- de las políticas implementadas?

\section{DISCUSIÓN DE LOS DATOS}

Inicialmente, llama la atención de cualquier analista el bajo grado de compromiso de la enseñanza superior con la igualdad. Si consideramos la promulgación de la ley de 2007 como punto de inflexión en las políticas de derechos paralelos, el impulso inicial académico fue el primer Encuentro de Unidades y Oficinas de Igualdad, que se celebró en enero de 2008 en la Universitat de les Illes Balears con la asistencia de representantes de 13 instituciones. En este momento, transcurridos cinco años desde el impulso legislativo, 21 universidades destacan los planes de igualdad en sus páginas web, de ellas 3 privadas.

En segundo término, más allá de un conjunto de datos estadísticos sobre la desigualdad horizontal y vertical segmentados por género en los distintos colectivos que integran la comunidad universitaria, la mayoría de los planes carecen o no mencionan haber realizado 
un diagnóstico científico de la igualdad en su institución. De los planes consultados, únicamente 7 universidades incluyen conceptos científicos relativos a la igualdad, incluso se omite la palabra "social" y es evidente que solo se puede ser igual o desigual respecto a variables sociales: poder, clases sociales, estratificación social, género... Pese a que no se localizan conceptos y referencias teóricas y/o empíricas relativas a la igualdad y al género, se ha considerado la inclusión de la idea de "transversalidad-mainstreaming" en 17 universidades como "palabra clave" para identificar la voluntad de estimar estos temas, aunque sea de forma intuitiva. Concluido el diagnóstico, en la secuencia lógica de un plan, se prosigue con la descripción de las acciones que deben ser implementadas. En este paso, todas las universidades en las que se ha reconocido la existencia de un plan presentan un programa de trabajo hacia la igualdad, pero es innecesario advertir que éstas acciones deben estar acompañadas de instancias responsables de llevarlas a cabo y de recursos humanos y económicos, que solo muestran 12 instituciones de enseñanza superior. Por otra parte, cuando estas medidas afectan a los derechos y deberes laborales, a las condiciones de trabajo, a las posibilidades de estabilidad y promoción, etc... estamos hablando de una normativa que, por su importancia, debería aplicarse con la mayor transparencia y responsabilidad posible. Por tanto, conforme con la ley, los planes deben contar con la participación de los trabajadores o al menos con la representación democrática de los mismos en los sindicatos, circunstancia que se indica en 8 de los planes. Definitivamente, todo plan debe ser evaluado para conocer el grado de desarrollo de las políticas, un final de proceso que es cumplido por 9 de las universidades.

\section{REFLEXIONES FINALES}

A luz de los hallazgos de la investigación, debe concluirse que la igualdad es un tema menor para las universidades españolas. El número de planes visualizados es exiguo y, sobre todo, las frecuencias que indican cumplimiento en los distintos apartados son desalentadoras respecto a la atribución que, comúnmente, se hace a la enseñanza superior de vanguardia y compromiso social. Además, el criterio de visibilidad -un concepto capital en las ciencias sociales y en el pensamiento feminista-, se revela trascendental en estas conclusiones, porque nos va a permitir descubrir datos latentes -las omisiones, inconscientes o conscientes- que, también, incluyen los planes.

En primer lugar, estos datos latentes manifiestan que las autoridades del Ministerio, las agencias de evaluación y los equipos de gobierno académicos han priorizado la participación del profesorado en el Espacio Europeo de Educación Superior (EEES) sobre el mandato constitucional de la igualdad. Como precisó (Bourdieu, 2003), en el mundo científico orientado por la competencia en búsqueda de beneficios específicos, las actividades del profesorado pueden ser definidas como desinteresadas, pero solo operan mediante mecanismos de recompensas. Y, es evidente, que los estímulos -vicedecanías, facilidades para acreditar al profesorado o las actividades formativas sobre competencias subvencionadas por entidades particulares-, se han dirigido las recompensas a los partícipes del proceso de Bolonia, pero no a quienes trabajaban por la igualdad.

Asimismo, tras promulgarse la Ley de 2007 las enseñanzas superiores debieron adaptarse a los criterios del EEES para alcanzar la verificación de la ANECA. Las conclusiones del trabajo de (Pastor Gosálbez, Belzunegui Eraso, Moreno Trigu, Mañas Rodríguez, 2010) sobre la Universitat Rovira i Virgili y las referencias de algunas 
universidades a las asignaturas que incluyen contenidos transversales en igualdad y género son axiomáticas. Pocas materias acogen estos temas obligatorios $\mathrm{y}$, además, están agrupadas en las ciencias sociales y humanidades. A la espera de más estudios, es necesario interrogarse por las carencias en otras áreas y, sobre todo, por los procedimientos por los que fueron aprobadas las nuevas enseñanzas, tanto por el profesorado, como por las Facultades, Departamentos, Vicerrectorados correspondientes y por la ANECA. Y, definitivamente, si los nuevos programas formativos del EEES pueden continuar reproduciendo un currículum oculto caracterizado por, entre otros valores, el androcentrismo.

Respecto al carácter científico de los planes, se precisa reconocer su construcción social a través de la vía emprendida por un primer núcleo de profesorado comprometido con la igualdad y el género, cuyas actividad docente e investigadora se sitúa en las áreas que tienen por centro estos temas de estudio. Validados marcos teóricos y efectuadas investigaciones, este grupo académico trabajó con movimientos sociales y, asimismo, consiguió el beneplácito de sus pares universitarios, con la inclusión de asignaturas, enseñanzas propias y, por último, fueron reconocidas como las personas más capacitadas para elaborar los planes de igualdad. Por estos motivos, los hallazgos de la investigación muestran una correlación casi perfecta entre las universidades pioneras y los planes redactados conforme a criterios científicos. Es evidente que no puede improvisarse ningún tipo de saberes y, con la certeza que muestran los datos, más allá del reducido número de universidades que iniciaron sus pasos hacia la igualdad a instancias de las labores de su profesorado, pocos avances pueden ser localizados en otras instituciones educativas, que se han limitado a cumplir de forma administrativa la ley o a realizar planes más cercanos a la empresa que a la universidad.

Es resaltable que todos los planes examinados presenten un programa de trabajo hacia la igualdad, aunque como se ha comprobado no todos parten de un diagnóstico, ni de una formulación científica. Además, muchos de ellos revelan la inexistencia de responsables de las acciones, la carencia de recursos humanos y de dotaciones económicas, un déficit democrático al obviar a la representación legal de los distintos colectivos universitarios y, por último, ningún mecanismo para evaluar cómo se han implementado las políticas igualitarias. Debería ponerse en consideración de la comunidad científica, que un documento redactado sin estas cualidades, puede ser una mera translación de postulados legales, administrativos y/o empresariales, pero, con dificultad, puede ser caracterizado como un plan de igualdad universitario.

Los planes constatan, a través de las estadísticas obligatorias, la segregación horizontal y vertical de género. Pero este diagnóstico, posiblemente, no es suficiente para alcanzar la igualdad. Por estas limitaciones, sin un riguroso conocimiento de las desigualdades sociales que acontecen en el seno de la universidad, la hagiografía de los burócratas ministeriales, las agencias evaluadoras y los directivos universitarios siguen enfatizando una serie de creencias sobre el conjunto del profesorado -libertad, flexibilidad y autonomía-, para justificar la aplicación de reglas "neutrales", basadas en el "mérito universal" (Gill, 2009). Por supuesto, como advertía hace tiempo (Alberdi, 1996) y se ha demostrado en la investigación, las discriminaciones sutiles presentadas como hábitos y costumbres "naturales" son las más difíciles de combatir. Aún, todavía, algunos tecnócratas desconocen o niegan los conceptos corrientemente admitidos en las ciencias sociales y en el feminismo de relaciones de poder, clases sociales, mecanismos informales de reproducción a través de fraternidades masculinas y religiosas $\mathrm{y}$, definitivamente, el 
peso de la herencia cultural, el patriarcado y los trabajos de cuidados como determinantes de las desigualdades en la universidad, sin contemplar medidas de corrección frente a las mismas.

Un continuismo descrito reiteradamente en las ciencias sociales y que para los ideólogos del poder parece agotarse, en palabras de Beauvoir (2008), "en una filosofía de la inmanencia que condena todo proyecto". Para cualquier persona estudiosa de la igualdad, las ciencias sociales y las teorías feministas tienen un desarrollo científico suficiente para que, ante la inacción de los poderes académicos y sus consecuencias sobre las mujeres y las sociedades, se consideren otras vías.

No puede concluir esta investigación sin recordar que los pensamientos más conocidos de Beauvoir, Bourdieu o Woolf han quedado reflejados en este texto, pero las limitaciones de páginas y caracteres a los que debe someterse toda escritura, solo nos permitan apuntar algunas palabras clave y autoras, en una relación que no puede ser exhaustiva, como base para futuros trabajos cuyo propósito sea lograr la igualdad real en la educación: sororidad (Lagarde, 1989), cyborg (Haraway, 1995) y teoría queer (Butler, 2001 y Wittig, 2005).

\section{BIBLIOGRAFÍA}

ALBERDI, I. (1996). "Las acciones positivas y la igualdad de oportunidades", en Mujeres, 21, pp. 16-20.

ALGUACIL, J. (2012), "La quiebra del incompleto sistema de Servicios Sociales en España", en Cuadernos de Trabajo Social, $\mathrm{n}^{\circ} 25$ (1), pp. 63-74.

APPLE, M. (1986), Ideología y Currículo. Madrid: Akal.

BEAUVOIR, S. (2008), El pensamiento politico de la derecha. Huelva: Doble J.S.L.U.

BERNSTEIN, B. (1988), Clases, códigos y control. Madrid: Akal.

BOURDIEU, P. (1999), "Sin movimiento social no hay política social", en Revista de Sociología UNMSM. Facultad de Ciencias Sociales, Vol 11, $\mathrm{n}^{\mathrm{o}}$ 12, en http://sisbib.unmsm.edu.pe/ BibVirtual/Publicaciones/sociologia/1999_n12/art021.htm, visitado 12/06/2012.

BOURDIEU, P. (2000), La dominación masculina: Barcelona: Anagrama.

BOURDIEU, P. (2003), Capital cultural, escuela y espacio social. México: Siglo XXI.

BOURDIEU, P. (2004), "Las estrategias matrimoniales en el sistema de las estrategias de reproducción", en Bourdieu, P., El baile de los solteros. Barcelona: Anagrama, pp. 167-210.

BOURDIEU, P. y PASSERON, J. C. (1985), La reproducción. Barcelona: Laia.

BOURDIEU, P. y WACQUANT, L. (1995), Respuestas. Por una Antropología Reflexiva. Barcelona: Grijalbo.

BUTLER, J. (2001), El género en disputa: el feminismo y la subversión de la identidad. Barcelona: Paidós Ibérica.

CALLEJO, J. (2004), El techo de cristal en el sistema educativo español. Madrid: Universidad Nacional de Educación a Distancia.

CANINO, M.V, y VESSURI, H. (2008), "La universidad en femenino. Un cuadro de luces y sombras en la UCV", en Arbor Ciencia, Pensamiento y Cultura CLXXXIV, n 733 septiembreoctubre, pp. $845-861$.

CARLOS, M.A. (2007), "Educación y Género", en Educación Superior, año VI, n 1 y 2, pp. 83-92.

CHOMSKY, N. y FOUCAULT, M (2006), La naturaleza humana: justicia versus poder. Un debate. Buenos Aires: Katz.

COBO, R. (1991), Las mujeres españolas: lo privado y lo público. Madrid: CIS.

COBO, R. (2011), Hacia una nueva política sexual. Las mujeres ante la reacción patriarcal. Madrid: Los libros de la catarata. 
COLLINS, R. (1979), La sociedad credencialista. Sociología histórica de la educación y de la estratificación. Madrid: Akal.

CRUZ, L. y SANZ, L. (2010), Endogamia, Productividad y Carreras Académicas. Instituto de Políticas y Bienes Públicos (IPP), CCHS-CSIC, Documento de Trabajo, $\mathrm{n}^{\circ}$ 1, en http://hdl.handle .net/10261/24401, visitado 14/05/2012.

DÍEZ GUTIÉRREZ, E.J. (2009), "El capitalismo académico: la reforma universitaria europea en el contexto de la globalización", en Revista Iberoamericana de Educación n. ${ }^{\circ}$ 50/1, en http://www.rieoei.org/jano/2904Diez-JANO.pdf, visitado 20/05/2012.

DURÁN, Ma . A. (1996), "El trabajo invisible en España: aspectos económicos y normativos”, en Documentación social, ${ }^{\circ}$ 105, pp. 137-158.

DURÁN, Ma ${ }^{\text {a }}$ A. (1999), Los costes invisibles de la enfermedad. Bilbao: Fundación BBVA.

DURÁN, Ma . A. (2000), "La nueva división del trabajo en el cuidado de la salud", en Política y sociedad, $\mathrm{n}^{\circ} 35$, pp. 9-30.

DURÁN Ma . A. (2006), "Dependientes y cuidadores: el desafío de los próximos años”, Revista del Ministerio de Trabajo e Inmigración, $\mathrm{n}^{\circ}$ 60, pp. 57-74.

ELIZONDO, A; NOVO, A. y SILVESTRE, M. (2010), "Igualdad de mujeres y hombres en las universidades españolas", Trabajo presentado en el Congreso Internacional: "La políticas de equidad de género en prospectiva: nuevos escenarios, actores y articulaciones". Área Género, Sociedad y Políticas- FLACSO- Argentina, noviembre, 2010.

ESPING-ANDERSEN, G. (1990), The Three Worlds of Welfare Capitalism, Oxford, Polity Press.

EUROSTAT (2009), The provision of childcare services: A comparative review of 30 European countries, Luxembourg, Office for Official Publications of the European Communities.

FERNÁNDEZ, L. (2008), “Género y ciencia: ¿Paridad es equidad?”, en Arbor Ciencia, Pensamiento y Cultura CLXXXIV 733, pp. 817-826.

GARCÍA DE LEÓN, Ma . A. (1990), "Las profesoras universitarias: el caso de una élite discriminada", en Revista Complutense de Educación, vol. 1, nº 3, pp. 355-372.

GARCÍA DE LEÓN, Ma. A. (2001), Las académica,s profesorado universitario y género. Madrid: Instituto de la Mujer.

GILL, R. (2009), "Breaking the silence: The hidden injuries of neo-liberal academia" en Flood, R. y Gill, R. (eds.), Secrecy and Silence in the Research Process: Feminist Reflections, London, Routledge, en https://www.kcl.ac.uk/artshums/depts/cmci/people/papers/gill/silence.pdf, visitado 11/05/2012.

GOLDSTEIN, L. F. (1982), "Feminist themes in French utopian socialism: The St. Simonians and Fourier", en Journal of the history of ideas, vol. 43, $\mathrm{n}^{\mathrm{o}} 1$, pp. 91-108.

GONZÁLEZ, M. J. y JURADO, T. (2009), “¿Cuándo se implican los hombres en las tareas domésticas? Un análisis de la Encuesta de Empleo del Tiempo", en Revista Panorama Social, 65-81. HARAWAY, D. (1995), Manifiesto para cyborgs. Valencia: Episteme.

INGLEHART, R. (1977), The Silent Revolution. Changing Values and Political Styles Among Western Publics. Princeton: Princeton University Press.

IZQUIERDO, Mª J. (2004), El sexisme a la UAB Propostes d'actuació i dades per a un diagnòstic. Bellaterra: Universitat Autònoma de Barcelona, Servei de Publicacions.

LAGARDE, M. (1989), Los cautiverios de las mujeres: madresposas, monjas, putas, presas y locas. México: UNAM.

MORALES, M. J.; LUNA, Mª J. y ESTEBAN, A. I. (2010), "Diagnóstico de paridad en la universidad: análisis a través de indicadores", en Revista de Universidad y Sociedad del Conocimiento, vol. $7 \mathrm{n}^{\circ}$ 2, en http://rusc.uoc.edu/ojs/index.php/rusc/article/view/v7n2-morales-lunaesteban, visitado 19/05/2012.

MORÁN, M. L. (2011), "La cultura política de las mujeres un campo de estudio todavía por explorar", en Psicología Política, n ${ }^{\circ} 42$, (Ejemplar dedicado a: Mujer y política en la España del siglo XXI), pp. 45-68.

NAVARRO, V. (2008), "Las políticas sociales de la socialdemocracia en la UE-15 y en España", en Temas para el debate, $\mathrm{n}^{\circ} 159$ (Ejemplar dedicado a: Avances en el Estado de Bienestar), pp. 30-33. PARKIN, F. (1984), Marxismo y teoría de clase. Una crítica burguesa. Madrid: Espasa Calpe. 
PASTOR, I.; BELZUNEGUI, A.; MORENO, B. y MAÑAS, C. (2010), "La igualtat d'oportunitats a la universitat: les percepcions del PDI", en Papers, n $^{\circ}$ 95/2, pp. 457-481.

PATEMAN, C. (1995). El contrato sexual. Barcelona: Anthropos.

POVEDA, M. M. (2008), "Desprovistas de poder pero nunca olvidadas: las desigualdades de género como elemento estructurante de la organización del trabajo", en Arxius de Ciències Social, no 19, pp. $115-128$.

PUJOL, H. y ORTIZ, A. (2009), “Acadèmia i igualtat de gènere: ¿només un miratge?”, en Documents d'anàlisi geogràfica, $\mathrm{n}^{\circ}$ 55, 129-145.

SÁNCHEZ BELLO. A. (2002), "El androcentrismo científico: el obstáculo para la igualdad de género en la escuela actual", en Educar, n 29, pp. 91-102.

SHOWSTARKC, A. (ed.) (1996), Las mujeres y el estado. Madrid: Vindicación Feminista.

WITTIG, M. (2005), El pensamiento heterosexual y otros ensayos. Madrid: Berkana.

WOOLF, W. (2008), Una habitación propia. Barcelona: Seix Barral.

\section{Breve currículo:}

\section{Francisco de los Cobos Arteaga}

Doctor en Sociología por la Universidad Complutense de Madrid y profesor titular de la Universidad de Castilla-La Mancha, ha centrado sus investigaciones en el mundo del trabajo y en las relaciones laborales. Sus últimas publicaciones han sido en la obra colectiva "Organizaciones obreras y represión en el ferrocarril: una perspectiva internacional" y "Los trabajadores de los Talleres Generales de la Compañía de los Ferrocarriles de Madrid a Zaragoza y Alicante", en la revista Sociología del Trabajo. En estos momentos, estudia el trabajo oculto de las mujeres en la industria. 